\title{
Impact of magnetic nanoparticle surface coating on their long-term intracellular biodegradation in stem cells
}

\begin{abstract}
Anouchka Plan Sangnier ${ }^{\dagger, \ddagger}$, Aurore B. Van de Walle ${ }^{+*}$, Alberto Curcio ${ }^{\dagger}$, Rémi Le Borgnell, Laurence Motte $^{\ddagger}$, Yoann Lalatonne ${ }^{\ddagger}, \S *$, Claire Wilhelm ${ }^{\dagger} *$

Magnetic nanoparticles (MNP) internalized within stem cells have paved the way for remote magnetic cell manipulation and imaging in regenerative medicine. A full understanding of their interactions with stem cells and of their fate in the intracellular environment is then required, in particular with respect to their surface coatings. Here, we investigated biological interactions of MNP composed of an identical magnetic core but coated with different molecules: phosphonoacetic acid, polyethylene glycol phosphonic carboxylic acid, caffeic acid, citric acid, and polyacrylic acid. These coatings vary in the nature of the chelating function, the number of binding sites, and the presence or absence of a polymer. The nanoparticles magnetism was systematically used as an indicator of their internalization within human stem cells and of their structural long-term biodegradation in a 3D stem cell spheroid model. Overall, we evidence that the coating impacts the aggregation status of the nanoparticles and subsequently their uptake within stem cells, but has little effect on their intracellular degradation. Only a high number of chelating functions (polyacrylic acid) had a significant protective effect. Interestingly, when the nanoparticles aggregated prior to cellular internalization, a lower degradation was also demonstrated. Finally, for all coatings, a robust dose-dependent intracellular degradation rate was demonstrated, with higher doses of internalized nanoparticles leading to lower degradation extent.
\end{abstract}

\section{Introduction}

Magnetic nanoparticles (MNP) are generating a growing interest in biomedicine as a result of their multifunctional features and tuneable properties. ${ }^{1}$ Several types of magnetic nanoparticles are already approved by the food and drug administration (FDA), ${ }^{2}$ used for example for iron supplementation in case of anemia. Pre-clinical and clinical studies also include applications such as targeting and drug delivery, ${ }^{3-5}$ gene therapy, ${ }^{6}$ thermal cancer therapies, ${ }^{7-9}$ or regenerative medicine. For this latter case, they are used to monitor stem cells implantation on site by MRI. ${ }^{10-13}$ Alternatively, they can be used to manipulate stem cells at a distance thanks to a remote stimulation, an original tool in the development of tissues ${ }^{14,15}$ or for cell targeting. ${ }^{16,17}$ Besides, and fundamental for all healthcare applications, iron oxide nanoparticles show good biocompatibility given by their iron core composition that can integrate the organism iron metabolism. ${ }^{18,19}$

For most regenerative medicine applications, MNP are internalized into stem cells and then left within, for purposes of manipulation or MRI imaging. To maintain MNP stability in aqueous environment, they are typically coated with molecules anchored to their surface via a chelating function. ${ }^{20-22}$ Among available iron chelating agents, citric acid is one of the most used coating agent in biomedical applications. ${ }^{23-25}$ This last decade, phosphonate and catechol also appeared amongst effective iron chelating agents. ${ }^{26-30}$ Additionally, besides improving water solubility, the terminal carboxylic acid function of these chelating agents can be used for later postfunctionalization, leading to targeted therapies. ${ }^{31-34}$

The surface coating is also considered a key parameter influencing the efficiency of the nanoparticles internalization with cells. For instance, negatively charged nanoparticles, as opposite to neutral ones, are known to successfully interact with cells. $^{35}$ The coating also influences nanoparticles interactions with their environment before being endocytosed by cells. Indeed, the surface of nanoparticles intravenously injected is immediately covered by a dynamic layer of blood proteins, known as the protein corona. ${ }^{36-40}$ The development rate and composition of the corona varies depending on the nature of the coating. ${ }^{40-43}$ Charged or hydrophilic nanoparticles are more likely to create stable interactions with proteins than hydrophobic ones. ${ }^{44}$ For instance, some coatings, such as polyethylene glycol (PEG), which is hydrophilic but not charged, provides stealth to the nanoparticles and impedes the anchoring of serum proteins. ${ }^{41}$ Nanoparticles are then known to be internalized together with their corona within cells before being trafficked to lysosomes and degraded. ${ }^{40}$

What is still to be unravelled is the impact that the nanoparticles coating may have on their structural fate, after internalization, within the intracellular environment. One limitation that magnetic nanoparticles can face for regenerative medicine applications is the risk of being quickly degraded in the lysosomes upon internalization, thus precluding any long-term imaging or magnetic stimulation. As a consequence, measuring how much nanoparticles are processed in the intracellular environment is crucial in order to develop biocompatible and potentially long lasting MNP. Most studies on nanoparticles degradation are reported in acid aqueous solutions containing iron chelating agents to mimic the lysosomal environment. ${ }^{45,46}$ Nevertheless, the intracellular environment is difficult to mimic due to the vast number of influencing factors such as $\mathrm{pH}$, oxygen tension, enzymes, proteins, and others, making in a way these models far from reality. On the other end, in vivo studies represent the highest degree of biological complexity, but this complexity makes it impossible to quantify the extent of degradation for molecules screening. Moreover, in the case of iron oxide nanoparticles, endogenous iron is present in the tissues and can be difficult to differentiate from nanoparticles. ${ }^{46}$ In between the two situations, an alternative 
choice to quantitatively monitor nanoparticles long term biodegradation is provided by tissue-mimicking 3D spheroids made of human mesenchymal stem cells (hMSC). ${ }^{47}$ Magnetometry measurements, commonly used to characterize nanoparticles upon their synthesis, ${ }^{48}$ performed on these confined tissues enable the precise quantitative study of the nanoparticles integrity over time.

Herein, we studied the cellular uptake and biodegradation of a set of iron oxide nanoparticles formulations that differ only by their coating, using an hMSC spheroid model established as a unique tool to monitor biodegradation. ${ }^{47,49,50}$ Starting from the same nanoplatform, we focused on the role played by (i) the coatings chelating function by using different molecules such as citric acid (carboxylic), caffeic acid (catechol) and phosphonoacetic acid (phosphonate), (ii) the number of chelating functions ( 3 vs 13 carboxylic-groups for citric acid and polyacrylic acid, respectively), (iii) the presence of a PEG spacer between the surface-binding function and the terminal external carboxylic group (- $\mathrm{COOH}$ vs $-\mathrm{PEG}-\mathrm{COOH}$ ) and also (iv) the presence of serum proteins. First, we evidenced that the coating has a pivotal impact on the cellular uptake. The PEG chain reduced significantly the internalization, while citric and phosphonoacetic acid exhibited a massive uptake, which was due to aggregation, yet prevented through nanoparticles opsonisation by serum proteins. Whether the conditions or coatings, one common behaviour is emerging: the lowest the amount of initial MNP, the highest the degradation extent. This demonstrates that the biodegradation rate is highly dependent on the initial intracellular quantity. At any given internalized mass, the direct role of coating on the long-term intracellular biodegradation was much lower. Only a polymeric coating with a high number of chelating functions was able to substantially decrease the biodegradation rate.

\section{RESULTS}

\section{A set of four different coatings on the same magnetic core}

A batch of magnetic nanoparticles (MNP) was synthesized according to a sol-gel microwave assisted method as previously described. ${ }^{51}$ As shown in Fig. $1 \mathrm{~A}$, spherical nanoparticles were obtained with a diameter of $8.8 \pm 2.5 \mathrm{~nm}$. These MNP were further coated with four different types of molecules, leading to nanoparticles sharing the exact same magnetic core (Fig. 1B). Two coatings were obtained using the same phosphonate chelating function: either phosphonoacetic acid (PO) alone, or polyethylene glycol phosphonic carboxylic acid (PO-PEG). They thus just differ by the PEG addition between the phosphonate chelating function and the carboxylic terminal group. Two other coatings were achieved, varying only the chelating function: caffeic acid (Caf) and citric acid (Cit). Importantly, all three chelating agents (PO, Caf, and Cit) exhibit the same terminal carboxylic acid function. Validation of each MNP coating was qualitatively and quantitatively assessed by Fourier Transformed Infrared measurements, zeta potential measurement and thermogravimetric analysis (Figures S1 and S2). Whatever the coating, MNP saturation magnetization was found at $51 \pm 2 \mathrm{emu} / \mathrm{g}_{\mathrm{Fe} 304}$. Stability was studied by dynamic light scattering (DLS, Fig. 1C) to monitor the evolution of MNP hydrodynamic diameter over time. Stability in RPMI medium alone was compared with RPMI medium supplemented with $10 \%$ foetal bovine serum (FBS) in order to mimic biological conditions with a layer of proteins attaching to the surface of the MNP. As a reference, hydrodynamic diameter (in intensity) obtained in water in physiological conditions $(\mathrm{pH}=7.4)$ was represented at the initial time and was determined around 50 $\mathrm{nm}$ for all coatings. In the case of MNP coated with PO-PEG (MNP@PO-PEG), with and without FBS, this diameter remained unchanged for at least 30 minutes, indicating stable MNP. For MNP coated with PO (MNP@PO) and with Cit (MNP@Cit), without FBS, the hydrodynamic diameter instantaneously increased to more than $1000 \mathrm{~nm}$, indicating a massive aggregation. On the contrary, the presence of FBS prevented this aggregation phenomenon with a hydrodynamic diameter increasing to 200 nm for MNP@PO and 80 nm for MNP@Cit. This can be explained by the formation of a protein corona which stabilizes the nanoparticles, considering also that binding on iron with citrate is weaker than with phosphonate so easier to be exchanged with proteins. ${ }^{52}$ Finally, for MNP coated with Caf (MNP@Caf), the hydrodynamic diameter slightly increased to $70 \mathrm{~nm}$ with proteins and $110 \mathrm{~nm}$ without proteins, showing no massive aggregation in both cases. 

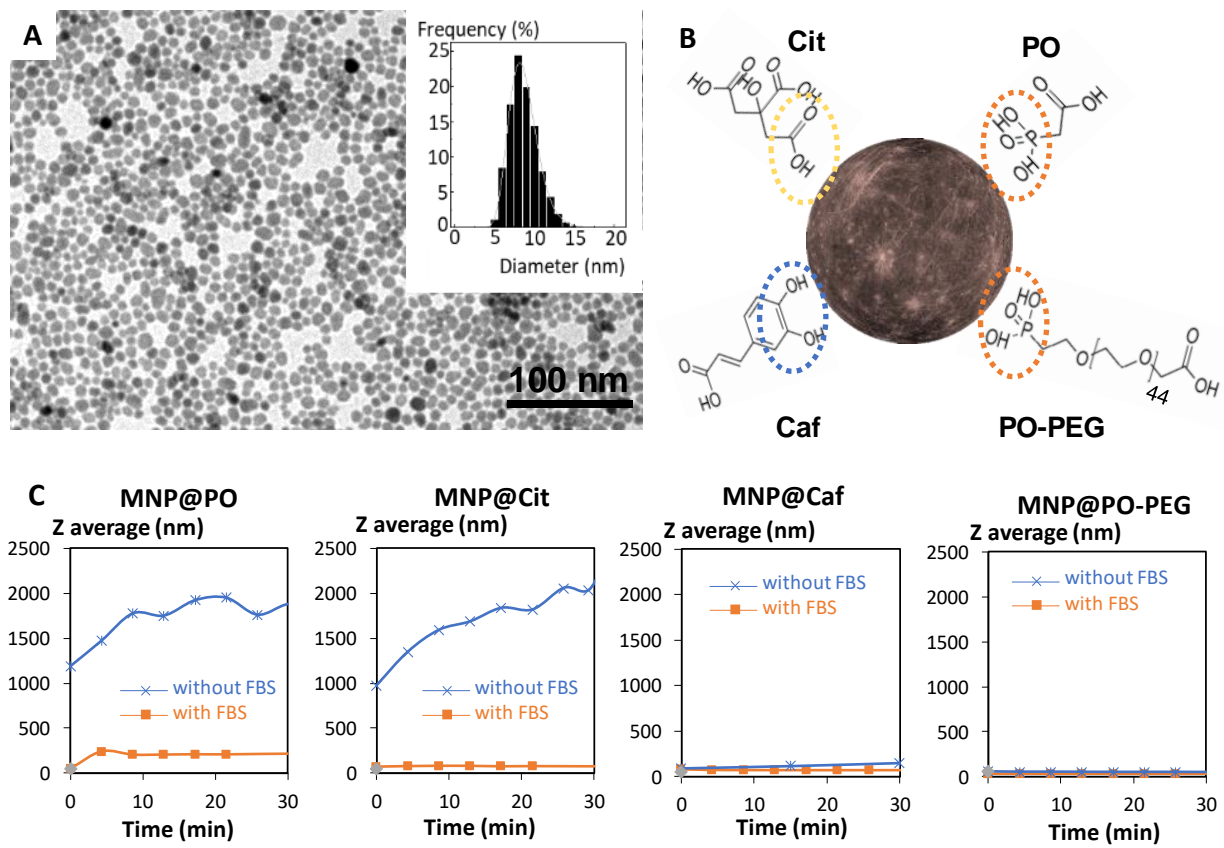

Figure 1: MNP panel characterisations. A. TEM imaging of non-coated iron oxide nanoparticles and associated size distribution. B. Schematic representation of coated magnetic nanoparticle with chemical formula of the selected coating molecules: PO, Cit, Caf, and PO-PEG. C. Hydrodynamic diameter represented as Z-average determined by DLS over 30 minutes, in RPMI medium without (blue curve) and with $10 \%$ FBS (orange curve), and at initial time in water at pH = 7.4 (grey point). From left to right: MNP@PO, MNP@Cit, MNP@Caf, and MNP@PO-PEG.

\section{Impact of the coating on cellular internalization}

To evaluate the immediate impact that the different coatings may have on their interaction with stem cells, hMSC were incubated with each MNP, in RPMI, with or without $10 \%$ FBS. MNP@PO, MNP@Cit, and MNP@Caf were incubated for 30 minutes, while MNP@PO-PEG needed to be incubated for 24 hours to detect an incorporation. MNP internalization in cells was quantified by magnetometry (Fig. 2A). Magnetization is directly proportional to the amount of MNP (magnetic iron oxide) in the sample (derived from the averaged saturation magnetization of the coated MNP, of $51 \pm 2 \mathrm{emu} / \mathrm{g}_{\mathrm{MNP}}$, see methods. As observed by us and others, the nanoparticle cell internalization increases with rising incubation concentration. For MNP@PO-PEG and MNP@Caf, saturation of intracellular content was reached at about $1 \mathrm{mM}$ incubation concentration, with an uptake saturation in between 5 and $10 \mathrm{pg}$ of MNP per cell, which did not depend on the presence of FBS in the incubation medium (with and without FBS conditions). The same uptake was obtained for MNP@PO and MNP@Cit when incubated with FBS. By contrast, without FBS, these two types of MNP exhibited a much higher cellular uptake than the other conditions, probably due to the aggregation of the nanoparticles evidenced in Fig. 1. Note however that MNP@POPEG needed to be incubated for 24 hours, meaning 48 times longer than the other MNP to reach similar internalization. The rate of internalization can be related to the surface charge of the MNP, quantified by assessing their zeta potential (Figure. S1).MNP@PO-PEG have a zeta potential that was found equal to $-10.8 \mathrm{mV}$, which explains their low internalization. This low charge suggests that some carboxylic groups could potentially be complexed on the MNP surface. On the contrary, zeta potentials of MNP@PO, MNP@Cit, and MNP@Caf were all substantially negative, respectively $-36,-39$, and $-33 \mathrm{mV}$, explaining the increased internalization. TEM imaging of the cells containing all types of MNP, one day after their internalization are shown in Fig. 2B, for the condition of incubation without FBS. They evidence the endosomal confinement of MNP, with no differences in the intracellular location observed depending on the coating. 

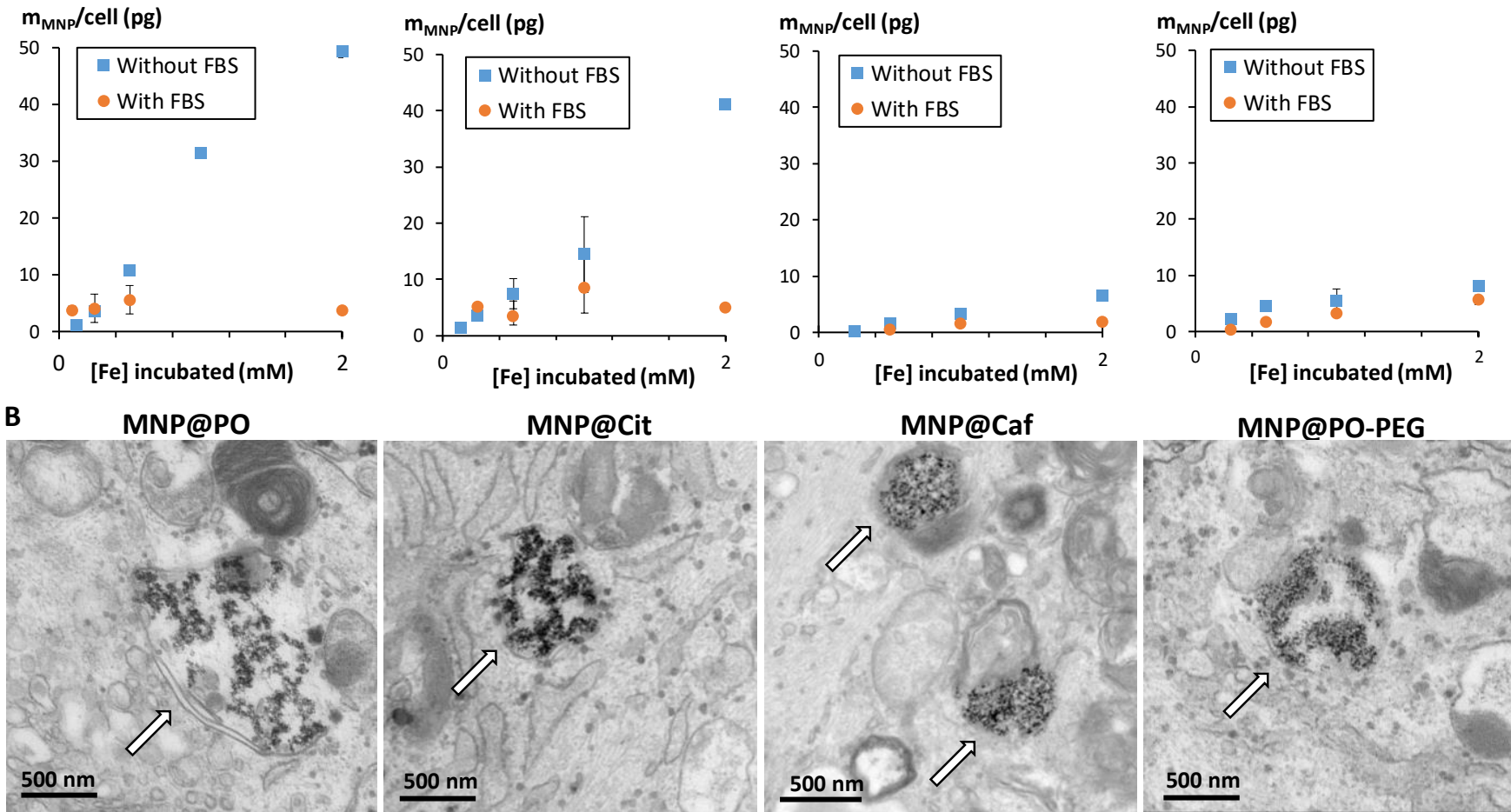

Figure 2: Coating impact on cellular uptake. A. Mass of MNP per cell after 30 minutes incubation for MNP@PO, MNP@Cit, and MNP@Caf and after 24 hours incubation for MNP@PO-PEG determined by magnetometry. B. TEM images of endosomes (arrows) within stem cells loaded with MNP@PO, MNP@Cit, MNP@Caf, and MNP@PO-PEG, all incubated without FBS.

\section{Impact on the intracellular degradation}

To assess the long-term transformations of the MNP inside the stem cells, hMSC having internalized each MNP (at various incubation dose but corresponding to an intracellular content of about $2.6 \mathrm{pg}$ of iron per cell) were assembled into cellular spheroids of 200000 cells and cultured for 21 days (Figure S3). Under the spheroid geometry, the stem cells stop dividing and produce a collagen-rich extracellular matrix leading to cellular spheroids viable for at least a month. ${ }^{47}$ They can be fixed (by glutaraldehyde) at different time (typically at days $1,4,8$, and 21), stopping instantaneously all active processes, including nanoparticles degradation. ${ }^{47}$ Single spheroid magnetization can then be measured at each time-point by magnetometry. Magnetization is directly proportional to the amount of MNP (magnetic iron oxide) in the sample, and as a consequence is the fingerprint of MNP structural integrity versus degradation. In order to obtain an accurate description of the biodegradation, five experiments were carried out with a total of around 900 spheroids formed for the four studied coatings (see methods). Fig. 3A shows typical magnetization curves for MNP@PO, at a given dose (corresponding here to 2.6 initial pg of iron per cell), and at different times of spheroids maturation. Magnetization was then converted in a mass of MNP, as shown in Fig. 3B, which decreases over time, indicating their intracellular degradation. Because the saturation magnetization can be affected by a change in the size of the nanoparticles, we first determined the nanoparticles diameter at the different degradation times (days 1, 4, 8 and 21). To do so, a Langevin analysis of the magnetization curves was performed, as detailed in Figure S4. Remarkably, at all times, the magnetic size matched perfectly the one of administered nanoparticles at day 1 . It evidences that the iron oxide core dissolution is rapid, and that intermediate states of partially degraded nanoparticles do not impact the magnetic measure. The magnetization measurement therefore provides a signature of the still intact nanoparticles only (with same size). The maximum loss in magnetization (equivalently to the maximum MNP degradation) is almost already reached at day 8 , and was found equal to $60 \%$ of the initial content for this condition. The same methodology was applied to all four coatings, for different initial intracellular MNP content (expressed in pg of iron per cell), and the degradation extent (obtained at day 21) was calculated for each. Figs. 3C, 3D, 3E and 3F summarize all data, for MNP@PO, MNP@Cit, MNP@Caf, and MNP@PO-PEG, respectively, and for incubations without or with FBS. Remarkably, whatever the condition, one same behaviour is evidenced: the lowest the amount of initial MNP, the highest the degradation extent. This demonstrates for the first time that the percent of MNP a cell can degrade depends on the initial intracellular quantity. 

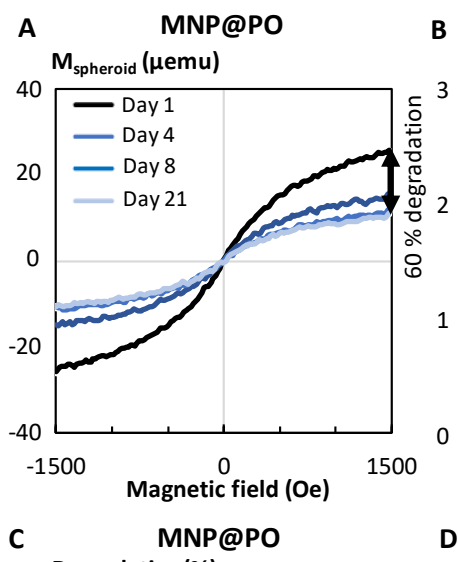

B MNP@PO Degradation(\%)

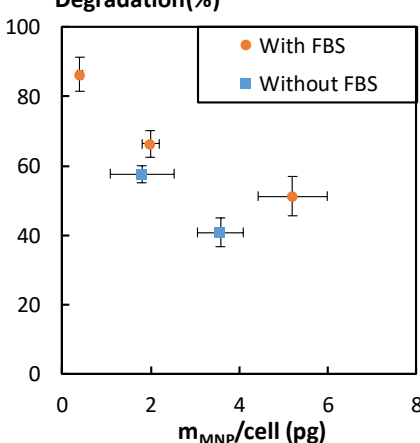
00 Degradation(\%)
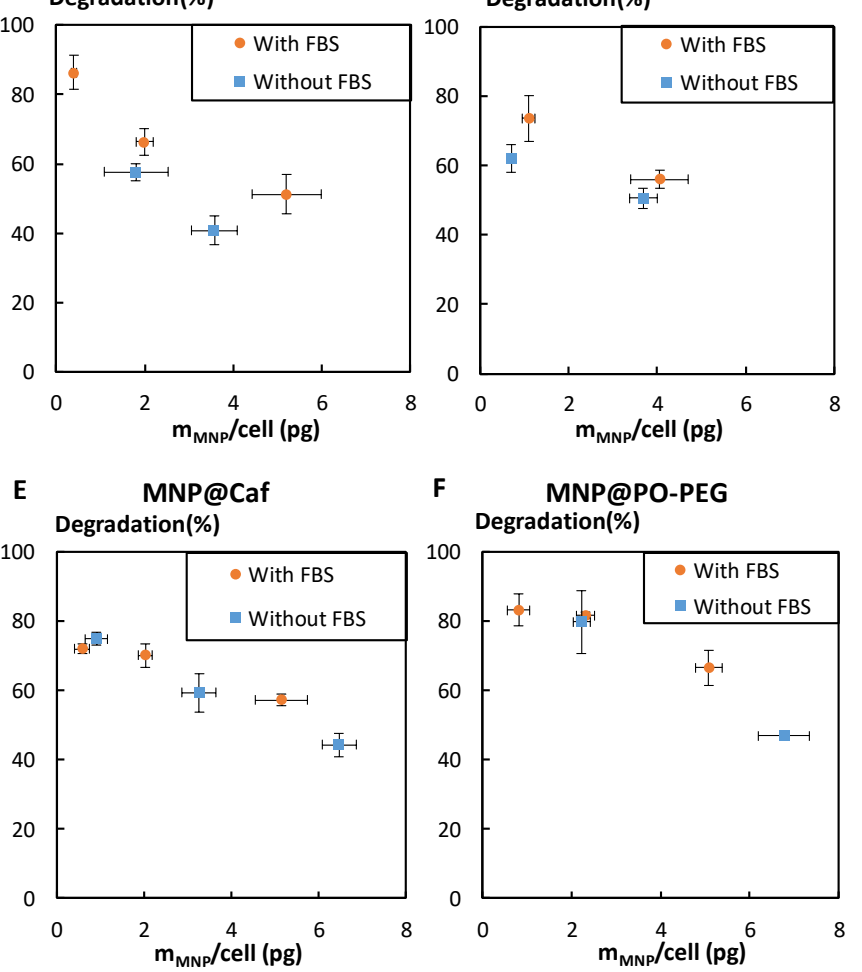

Figure 3: Coating impact on the intracellular degradation. A. Magnetization curves measured by magnetometry of hMSC spheroid loaded with MNP@PO (2.6 pg of iron per cell) at different times of spheroid maturation (days 1, 4, 8, and 21). B. Intracellular mass of MNP calculated from the magnetization values. C. Percentages of degradation of MNP@PO according to the intracellular mass of MNP D. Percentages of degradation of MNP@Cit according to the intracellular mass of MNP E. Percentages of degradation of MNP@Caf according to the intracellular mass of MNP F. Percentages of degradation of MNP@PO-PEG according to the intracellular mass of MNP.

While all conditions exhibit the same global behaviour, two groups emerge (Fig. 4). First, MNP@PO and MNP@Cit incubated without FBS, corresponding to the aggregated situation (Fig. 1C), experience the lowest rate of degradation (Fig. 4A). Secondly, stable nanoparticles, meaning MNP@PO and MNP@Cit, both with FBS; MNP@Caf and MNP@PO-PEG with and without FBS, exhibited a higher degradation (Fig. 4B). For each family, linear regression was plotted, with sound determination coefficient $\left(R^{2}>0.75\right)$, which confirmed the grouping of conditions and also the dose dependent degradation.
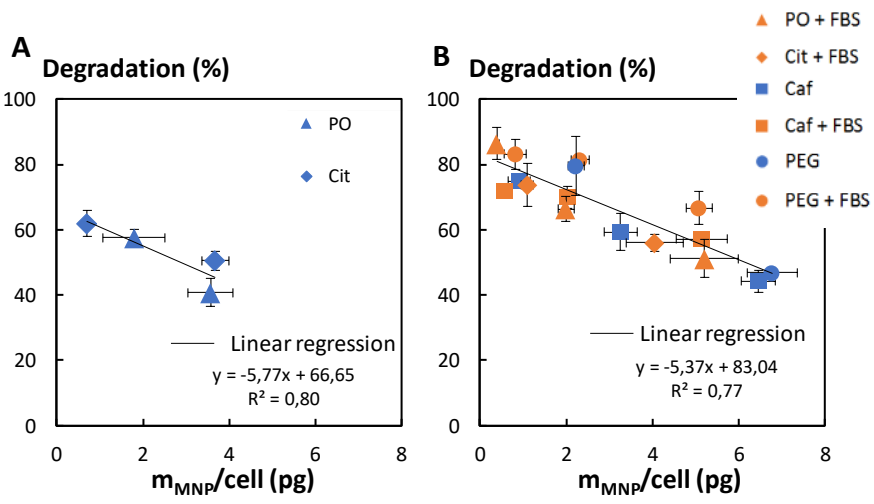

Figure 4: Percentages of degradation according to the intracellular mass of MNP by grouping the points into two families: A) The aggregated nanoparticles: MNP@Cit and MNP@PO without FBS. B) The stable nanoparticles: MNP@PO and MNP@Cit with FBS; MNP@Caf and MNP@POPEG with and without FBS. For each group, linear regression is plotted as a dotted line with its corresponding equation.

TEM imaging of the MNP confined within the cells endosomes (in the range of 3 pg of MNP per cell) 1 and 21 days after their internalization is shown in Fig. 5, for the condition of incubation without FBS (see also Figures S4 and S5). One day after spheroid formation MNP@PO and MNP@Cit looked more aggregated within the endosomes than MNP@Caf or MNP@PO-PEG, as expected from the stability curves of Fig. 1C. At day 21, a massive apparition of new structures in the endosomes and cytoplasm was revealed. They exhibit a circular shape and a diameter of $6.4 \pm 1.1 \mathrm{~nm}$, typical of ferritin. No difference was noticed between the different coatings, even for those which were likely to aggregate (MNP@PO and MNP@Cit). Those results are confirmed by large views TEM images presented in Figure S5 and in endosomes TEM images in Figure S6. In the case of the four coatings, some endosomes were still charged with intact MNP, corresponding to non-degraded MNP. In the case of MNP@PO and MNP@Cit in the absence of serum, these intact MNP were aggregated similarly to day 1 (Fig. 2B). For the other conditions, they also appeared the same, well dispersed within the endosomes. When MNP were incubated with FBS (Figure S7), all coatings appeared similar within endosomes, on TEM images, and exhibited the same apparition of ferritin when degraded. 

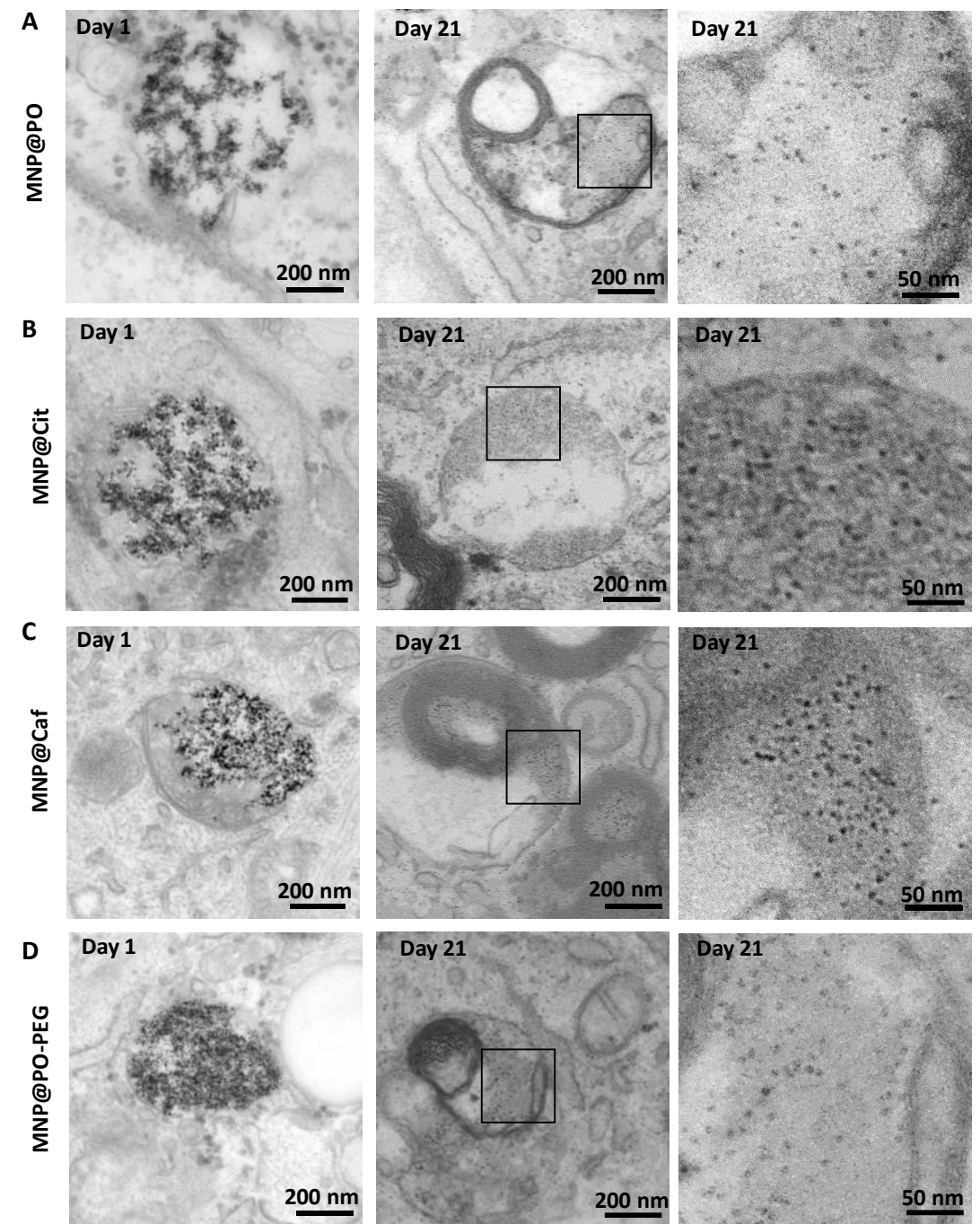

Figure 5: TEM images of endosomes 1 and 21 days after MNP internalization within cells for the different MNP incubated in the absence of FBS. For each coating, an endosome is represented 1 day after spheroid formation (left), then 21 days after spheroid formation (center) with an image zoom in the black square (right) to clearly identify the ferritin spots, for the different coatings: A. MNP@PO, B. MNP@Cit, C. MNP@Caf, and D. MNP@PO-PEG.

\section{A fifth coating, a polymer with multiple chelating functions}

Considering that the chelating agent had few impact on the MNP intracellular fate, we considered another coating strategy, using polyacrylic acid (PAA) (Fig. 6A), a polymer which binds to the MNP in several sites via its multiple $-\mathrm{COOH}$ function, in order to investigate its possible impact on degradation (such as a protective effect). Its successful binding to the surface of the MNP was assessed by FTIR, zeta potential and TGA, and the saturation magnetization of the MNP coated with PAA (MNP@PAA) found equal to 52 emu/g $\mathrm{g}_{\mathrm{Fe} 3 \mathrm{O}}$ (see Figures $\mathrm{S} 8$ and S9).MNP@PAA were found to be very stable with and without FBS (Fig. 6B) and internalized within cells in a dose-dependent manner, presenting the same tendency with and without FBS (Fig. 6C). MNP@PAA internalization is comparable to those of the previous non-aggregated nanoparticles: MNP@PO and MNP@Cit with FBS, MNP@Caf and MNP@PO-PEG with and without FBS (see Fig. 2A). Indeed, its surface charge is negative, with zeta potential of $-42.8 \mathrm{mV}$. The fact that FBS doesn't affect neither the stability nor the internalization of MNP@PAA suggests a protection provided by PAA from opsonisation with the proteins present in FBS. Fig. 6D shows the confinement of MNP@PAA within the endosomes the first day after internalization. The same methodology than for the other MNP was next followed for MNP@PAA to monitor biodegradation: MNP@PAA were internalized within the hMSC, 200000 cell spheroids were formed, and long-term quantification of nondegraded MNP was achieved by magnetization data capture (Fig. 6E) to assess degradation. Note that in this latter case, the intracellular dose corresponded to 3.1 initial pg of MNP per cell, which is similar to the intracellular dose used in Fig. 3B for MNP@PO. Here, for MNP@PAA, degradation was found to be of $40 \%$ for this dose, compared to 60\% for MNP@PO, suggesting a protective effect provided by PAA. Fig. 6F shows all intracellular doses further tested, ranging from 0.8 to $8 \mathrm{pg}$ of intracellular MNP per cell. Similar behaviour was observed with and without FBS. A dose-dependent effect was observed revealing again the most important degradation for the lowest concentrations, but the slope of the corresponding linear regression was lower than for the other MNP (Fig. 3F), preventing high amount of MNP@PAA to be degraded at low 
doses. TEM imaging of the cells 21 days after spheroid formation evidenced the presence of endosomes loaded with non-degraded MNP but also of the presence of ferritin corresponding to the degraded nanoparticles, also shown in Figure S10. The presence of proteins did not impact the endosomal confinement of MNP after cellular capture neither the apparition of ferritin corresponding to the degraded MNP after 21 days as evidenced in Figure S11.

A

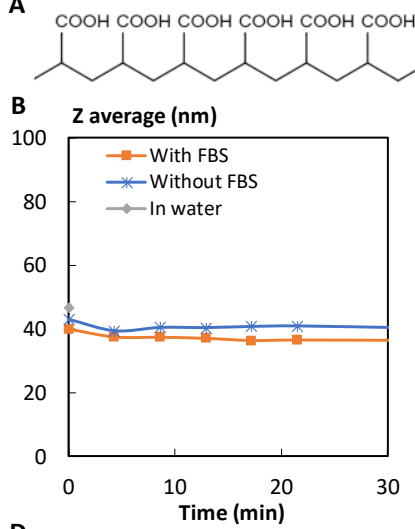

D

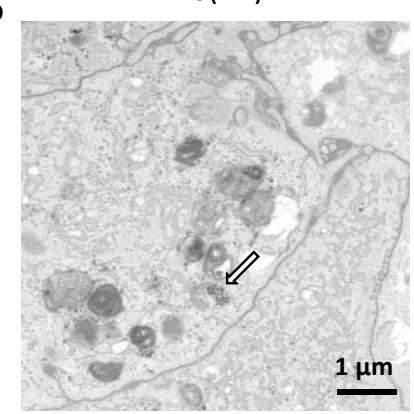

E

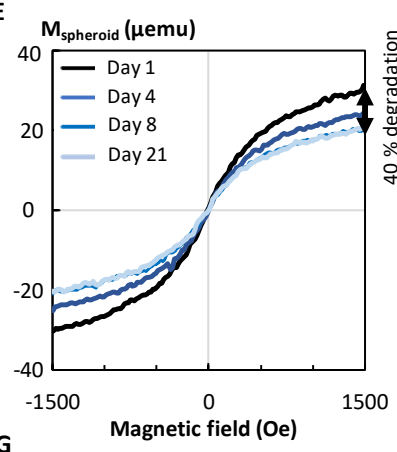

G
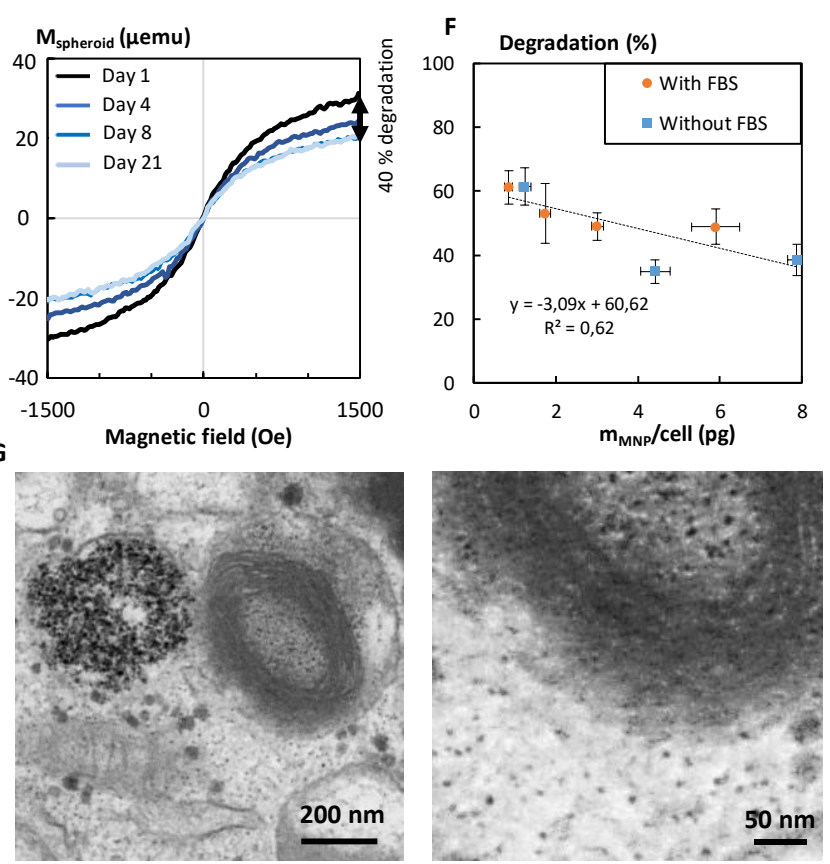

Figure 6: MNP@PAA characterisations, uptake and intracellular fate. A. PAA chemical formula. B. Hydrodynamic diameter represented as $z$ average was determined by DLS over 30 minutes, without (blue curve) and with $10 \%$ FBS (orange curve), and at initial time in water at $\mathrm{pH}=7.4$. C. Mass of iron per cell after 30 minutes incubation of the MNP without (blue) and without (orange) $10 \%$ FBS. D. TEM images of cells loaded with PAA without FBS 1 day after the spheroid formation. A large view is presented on the left and a zoom on a MNP loaded endosome on the right. E. Magnetization curves measured by magnetometry of hMSC spheroid loaded with PAA (3.1 pg of iron per cell) at different time of spheroid maturation (1, 4, 8 and 21 days). F. Percentages of degradation of MNP according to the intracellular mass of iron. G. TEM images of endosome loaded with PAA without FBS 21 days after the spheroid formation with an image zoom (right) to clearly identify the ferritin spots.

To manage a direct comparison in between MNP, it finally appeared necessary to compare them for the same initial intracellular dose. Figs. 7A and 7B were plotted to compare the degradation rate of all MNPs, incubated with and without FBS, leading to an intracellular iron concentration in the range of 2.4 $\pm 0.3 \mathrm{pg}$ of iron per cell. In presence of FBS, PAA was significantly less degraded than all the other MNP. In the absence of FBS, PAA was significantly less degraded than MNP@PO-PEG and MNP@Caf, but showed similar degradation than MNP@PO and MNP@Cit. This confirms the lower degradation of these MNPs observed in Figs. 3 and 4, reflecting their aggregation status. This was also observed in lysosome mimicking solution (Figure S12), in which no significant degradation differences were observed between all other types of MNP.
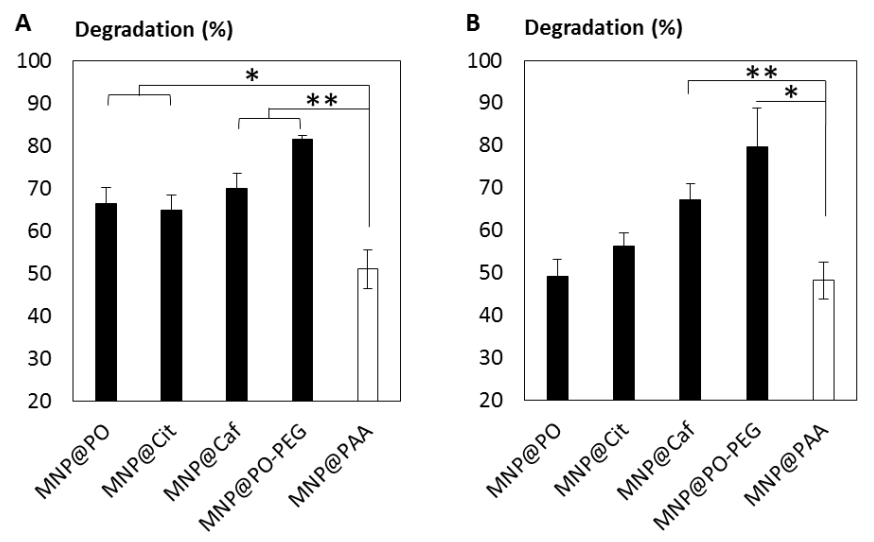

Figure 7: Comparison of the degradation of MNP@PAA with previously studied coatings. A. Comparison of the degradation percentage of PAA with the other MNP after an incubation of MNP with FBS according to the coating at the same dose of $2.4 \pm 0.3 \mathrm{pg}$ of MNP per cell, $* p<0.05$ and ${ }^{* *} \mathrm{p}<0.01$ between conditions (Student t.test). B. Comparison of the degradation percentage of PAA with the other MNP after an incubation of MNP without FBS according to the coating at the same dose of $2.4 \pm 0.3 \mathrm{pg}$ of MNP per cell, $* \mathrm{p}<0.05$ and $* * \mathrm{p}<0.01$ between conditions (Student t.test).

\section{Discussion}

Multiple studies were previously carried out to investigate the impact MNP design may have on cell uptake and especially focused on the role of the surface charge ${ }^{53}$. First, it is now generally accepted that charged nanoparticles exhibit a higher cellular uptake than neutral ones. ${ }^{54}$ Our results are consistent with the literature since MNP@PO-PEG, the one coating 
exhibiting a lower charge than the others in analysis, needed a 48-fold longer incubation time in order to reach similar intracellular iron intake. This result is also in accordance with other studies that define PEG as one of the best non-fouling agents, ${ }^{55-58}$ and that it also helps the nanoparticles to avoid immunological recognition of the mononuclear phagocyte system, reducing its clearance. Indeed, being a highly hydrophilic polymer, PEG forms a hydration layer leading to steric repulsion. ${ }^{59-61}$ As a consequence, PEG coating can be chosen to increase the circulation time in the blood of MNP injected intravenously. ${ }^{62}$ On the contrary, MNP@PAA, MNP@Caf, MNP@PO, and MNP@Cit showed a rapid internalization within cells. In the context of regenerative medicine, cells need to be efficiently labelled with the nanoparticles in vitro before being transplanted in vivo. ${ }^{12}$ Therefore, quickly internalized MNP can be seen as good candidates. Besides, we here evidenced a strong impact of the nanoparticles' stability on the cellular uptake: aggregated nanoparticles led to higher internalization within the cells than stable ones. This stability is influenced by the nature of the coating, but also by the presence of macromolecules in the incubation media, and the formation of a protein corona. The formation of the corona appears quickly enough to stabilize the MNP by steric stabilization and by preventing van der Waals attractive forces, ${ }^{44,63}$ as previously observed for MNP@Cit aggregating in phosphate buffer solution (PBS) but stable when the PBS was supplemented with FBS. ${ }^{64}$ Due to their longer chains PO-PEG and PAA induce steric repulsions which explain why MNP@PO-PEG and MNP@PAA are stable without proteins contrary to MNP@Cit and MNP@PO. Finally, for all coatings, the uptake occurred through the internalization of the nanoparticles within the endosomes, as expected from negatively charged MNP. ${ }^{65}$ No trace of MNP was found within the cytoplasm or other cellular compartments, excepting the endosomes, and we showed that aggregated MNP similarly ended in the endosomes.

After cellular internalization, MNP were degraded by the cells, as suggested by the decrease of cell magnetization and by the appearance of ferritin structures. Ferritin is the iron storage protein known to intracellularly store the iron released over nanoparticles degradation in a non-toxic form. ${ }^{66}$ Indeed, Fe(II) ions could react with hydrogen peroxide and produce cytotoxic free reactive oxygen species (ROS) through the Fenton reaction. Instead, if they are trapped within the ferritin, they are not available for Fenton reaction and thus non-toxic so ferritin is considered to be a ROS production inhibitor. ${ }^{66-68}$

The impact of the coating on the degradation of the MNP has been explored, but rarely in cells. A study conducted in lysosome mimicking solution comparing the degradation of MNP coated with citric acid or phosphonoacetic acid revealed that the firsts were less degraded than the latters. ${ }^{46}$ On the contrary, inside the cells, we obtained either no significant differences between those two coatings in the presence of FBS or a significant difference without FBS leading to the opposite result: MNP@PO were in fact less degraded than MNP@Cit. Degradation of MNP@PEG and MNP@glucose were also compared, indicating a faster biodegradation of MNP coated with glucose in lysosomal mimicking environment, but also a highly protective impact from the protein corona. On the contrary, in vivo, MNP@PEG were degraded quicker, possible consequence of the difficulty to mimic lysosomal environment but also the protein corona. ${ }^{69}$ Those results emphasize the need of in vitro models in order to mimic degradation pathways in biological-like conditions, a solution provided by hMSC spheroids. In vivo, while the coating is expected to strongly impact the nanoparticles interaction with blood proteins, and therefore their circulation, once in the intracellular environment, the nanoparticles fate is expected to be similar between the situation in vivo and in the spheroid model. To monitor the nanoparticles behavior in real-time in vivo, magnetic resonance imaging (MRI) should be used. Yet, a MRI signal fall is expected over their transformation from nanoparticles to ionic iron but this fall may be attenuated due to higher proximity of protons to the remaining nanoparticles, preventing precise quantification.

A factor that we found to have the strongest impact on the biodegradation is the internalized MNP dose. Indeed, the highest degradation rates were obtained for the lowest MNP concentrations. This result was also observed in vivo for MNP@PEG and MNP coated with DMSA. ${ }^{62}$ Finally, aggregation was also found to impair the MNP cell degradation (shown in the case of MNP@PO and MNP@Cit), ${ }^{46}$ which could be explained by less accessibility of degrading agents (protons, enzymes, etc.) at the core of aggregates.

Taken together, these results suggest the following picture: the coating of the nanoparticles has an immediate impact on its interactions with biological systems (fluids and cells). More precisely, surface charge plays an important role on the uptake, and the nature of the coating influences the stability of the nanoparticles in the biological media and interactions with biomolecules. Upon cellular internalization, increase of intracellular MNP dose reduces their degradation rate regardless of the coating. At a constant intracellular dose, slight modulations of the MNP degradation were observed between the coatings themselves. No impact is observed from the nature of the chelating function (carboxylic, phosphonate or catechol). The most significant impact lies on the protective effect of PAA towards degradation, even for small doses of intracellular MNP. PAA molecules, due to their multiple anchoring functions are more tightly bound to nanoparticles surface, probably remain for longer delaying the biodegradation. This tuning of the MNP degradation can be used according to the nature of the application requested, in order to optimize the clearance time of the nanoparticles from the tissues. In particular, one challenge in regenerative medicine is to develop long-lasting imaging agents. This study provides keys to better understand the in situ biodegradation of MNP but also emphasizes the variety and complexity of factors influencing it.

\section{Conclusions}

To conclude, coatings varying by the nature of surface anchoring functions turned out to have the same impact on the biodegradation. Only a higher number of anchoring functions 
(13 vs 3 , eg. PAA vs citrate) from the coating molecule to the surface of the MNP were impacting the biodegradation rate. Nevertheless, the different coatings have a strong impact on MNP colloidal stability in biological fluids, and aggregation was shown to reduce the biodegradation. It is important to pinpoint that the initial intracellular amount of MNP has a higher impact than the coating itself on controlling the nanoparticles biodegradation: the higher the dose is, the lower the degradation extent, down to $30-40 \%$ for our higher doses tested. In these conditions, up to $70 \%$ of MNP were left intact, which is overall very high in terms of magnetization, considering that it corresponds to high initial doses. Yet, for high doses, the question of toxicity stands, making MNP@PAA still the most promising, to provide a long-lasting cell magnetization with a reasonable doses of intracellular iron.

\section{Methods}

\section{MNP synthesis}

Iron(III) acetylacetonate (> 99.9\%) and Benzyl alcohol anhydrous (99.8\%) were purchased from Sigma Aldrich. MNP were synthesized using a procedure previously described..$^{50}$ $400 \mathrm{mg}$ of iron(III) acetylacetonate were dissolved in $10 \mathrm{ml}$ of benzyl alcohol. Then, MNP synthesis was carried out in a Monowave 300 from Anton Paar. The temperature of the suspension was increased up to $250^{\circ} \mathrm{C}$ in 20 minutes then maintained constant for 30 minutes. The resulting suspension was separated using a neodymium magnet, and the precipitate was successively washed with dichloromethane, sodium hydroxyde, ethanol, pH 7 water (three times for each except for ethanol only one washing step) then ultracentrifugated at $\mathrm{pH}=2$ in Amicon ${ }^{\circledR}$ Ultra centrifugal filters ( $\left.30 \mathrm{kD}\right)$. Finally, the $\mathrm{pH}$ of the suspension was adjusted at 2 .

\section{MNP coating}

Citric acid, poly(acrylic acid, sodium salt) (MW $1200 \mathrm{~g} / \mathrm{mol}^{-1}$ ), phosphonoacetic acid, and caffeic acid were purchased from Sigma Aldrich. PEG phosphonic carboxylic acid (SP-1P-10-002, MW $2500 \mathrm{~g} \mathrm{~mol}^{-1}$ ) was purchased from Specific Polymers. All the coatings were used in excess with a massic ratio of 5 between the coating molecule and the nanoparticles. Citric acid, polyacrylic acid, phosphonoacetic acid, and polyethylene glycol were diluted in water at $\mathrm{pH}=2$ and caffeic acid at $\mathrm{pH}=10$. The nanoparticles aqueous dispersion was added to the coating molecule solution and let for 2 hours under magnetic stirring. After the reaction, $\mathrm{pH}$ was adjusted at 7 and solution was let for 2 hours to allow for the system to equilibrate. Finally, $\mathrm{pH}$ was adjusted at 2 and nanoparticles were magnetically purified 3 times then ultracentrifugated at $\mathrm{pH}=7$ in Amicon $^{\circledR}$ Ultra centrifugal filters (30 kD). Nanoparticles coated with citric acid, polyacrylic acid, phosphonoacetic acid, caffeic acid, and PEG phosphonic carboxylic acid were respectively named MNP@Cit, MNP@PAA, MNP@PO, MNP@Caf and MNP@PO-PEG.

\section{MNP characterization}

The hydrodynamic diameter and the zeta potential of the nanoparticles were measured using a Nano-ZS (632.8 nm) ZEN 3600 device (Malvern Instruments, Malvern, UK). Nanoparticles were diluted in water at $\mathrm{pH}$ adjusted at 7.4 with $0.1 \mathrm{M} \mathrm{NaOH}$ to be representative of physiological conditions.

The successful presence of the coating of the particles at the surface of the molecules was checked by Fourier Transformed Infrared Spectroscopy (FTIR) using a Thermo Scientific Nicolet $380 \mathrm{FTIR}$. Samples were analysed on under the form of $\mathrm{KBr}$ pellets.

Quantification of coating molecules per particle was evaluated by TGA using a LabsSys evo TG-DTA-DSC 16000 device from Setaram Instrumentation.

The iron concentration of the nanoparticles was determined through a colorimetric quantification: iron was destroyed with $\mathrm{HNO}_{3}$ at $7 \mathrm{M} . \mathrm{H}_{2} \mathrm{O}_{2}$ was added to oxidize the iron ions. Then, iron concentration was determined by thiocyanate colorimetry.

The saturation magnetization of the nanoparticles $(51 \pm 2$ emu/g) was measured in a Vibrating Sampler Magnetometer (VSM, Quantum Design, Versalab) on $10 \mu \mathrm{l}$ of solution at $\left[\mathrm{Fe}_{3} \mathrm{O}_{4}\right]=1 \mathrm{mg} / \mathrm{ml}$. This magnetization in emu per $\mathrm{g}$ of $\mathrm{Fe}_{3} \mathrm{O}_{4}$ was used to convert magnetization values into mass of magnetic iron in a (cellular) sample.

\section{Cell culture and MNP internalization}

Human Mesenchymal Stem cells (hMSC) were purchased from Lonza. They were cultured in MSCBM medium (Lonza) at $37^{\circ} \mathrm{C}$ with $5 \% \mathrm{CO} 2$. At $90 \%$ confluency, cells were amplified until passage 5 before use. Nanoparticles were dispersed in RPMI1640 medium (ThermoFischer Scientific) or RPMI-1640 medium supplemented with $10 \%$ FBS at different iron concentrations. Cells were washed with RPMI then incubated with the nanoparticles solutions for 30 minutes in the case of MNP@Cit, MNP@Caf, MNP@PAA and MNP@PO and for 24 hours in the case of MNP@PO-PEG. Cells were washed with RPMI then media was changed to DMEM supplemented with $10 \%$ FBS and $1 \%$ Penicilin-streptomycin to allow a complete internalization of the nanoparticles ( 2 hours for MNP@PO-PEG, overnight for other nanoparticles).

\section{Spheroid model}

Cells loaded with nanoparticles were detached with TrypsinEDTA $(0.05 \%)$, phenol red (thermoFisher Scientifc), then 200000 cells were dispersed in $1 \mathrm{ml}$ of specific medium in $15 \mathrm{ml}$ Falcon ${ }^{\circledR}$ tubes before being centrifugated for 3 minutes at 180 $\mathrm{g}$ to form the pellet. Pellets were cultured at $37^{\circ} \mathrm{C}, 5 \% \mathrm{CO}_{2}$. The medium was replaced twice a week. Medium for spheroid maturation was composed of $500 \mathrm{ml}$ of DMEM high glucose supplemented with $1 \%$ penicillin streptomycin, $1 \%$ ITS premix and with a final concentration of $0.1 \mu \mathrm{M}$ dexamethasone, $1 \mathrm{mM}$ sodium pyruvate, $50 \mu \mathrm{M}$ ascorbic acid- 2 phosphate and 0.35 $\mathrm{mM}$ L-proline. Spheroids were washed with cacodylate buffer then fixed with $2 \%$ glutaraldehyde in $0.1 \mathrm{M}$ cacodylate buffer for 30 minutes at room temperature then stored in PBS in order to measure their magnetization at different days of the maturation process (days $1,4,8$, and 21 ). 


\section{Spheroid magnetometry}

Spheroids were introduced in sample holders for Vibrating Sampler Magnetometer (VSM, Quantum Design, Versalab). Magnetization curves according to the applied filed were recorded at $300 \mathrm{~K}$ in the range of 0 to $30000 \mathrm{Oe}$. The recorded magnetization was converted in grams of MNP in the sample using the saturation magnetization of $51 \pm 2 \mathrm{emu} / \mathrm{g}_{\mathrm{Fe}} \mathrm{OO4}$. Decrease of grams of MNP in the sample corresponded to the biodegradation of MNP.

Five independent experiments were carried out. For each coating, four doses were compared with triplicates for each point, and two (days 1 and 21) or 4 (days 1, 4, 8 and 21) were analysed, leading to a total of 220 individual spheroids per coating.

\section{Transmission Electron Microscopy of MNP and cells}

For transmission electron microscopy (TEM) imaging, nanoparticles $(10 \mu \mathrm{l})$ were deposited on carbon-coated copper grids then imaged with a Philips TECNAI 12 electron microscope. To observe MNP within the cells, spheroids were washed with cacodylate buffer, fixed with $2 \%$ glutaraldehyde in $0.1 \mathrm{M}$ cacodylate buffer. Then samples were contrasted with Oolong Tea Extract (OTE) $0.5 \%$ in cacodylate buffer, post fixed with $1 \%$ osmium tetroxide containing $1.5 \%$ potassium cyanoferrate then dehydrated in graded ethanol baths, included in Epon, and ultrasectioned $(70 \mathrm{~nm})$. Sections were deposited onto cooper grids for observation with a TECNAI 12 electron microscope.

\section{Degradation in aqueous suspension}

Degradation was assessed in a medium mimicking lysosomal conditions, as previously described. ${ }^{46} \mathrm{MNP}$ were diluted at 1 $\mathrm{mM}$ in $20 \mathrm{mM}$ citrate buffer solution at $\mathrm{pH}=4.7$ then kept at room temperature during 21 days. Magnetization was measured in a Vibrating Sampler Magnetometer (VSM, Quantum Design, Versalab) on $10 \mu$ l of solution. The decrease of magnetization of the sample corresponded to the degradation of the sample.

\section{Statistical Analysis}

All values are reported as mean \pm standard error of the mean (SEM). Significance between groups was determined using Student's t-test. Results were considered significant when $\mathrm{p}<0.05$ and noted $* \mathrm{p}<0.05$ and $* * p<0.01$

\section{Conflicts of interest}

There are no conflicts to declare.

\section{Acknowledgements}

This work was supported by the European Research Council (ERC-2014-CoG project MaTissE 648779). We also acknowledge technical support from CNanoMat-University Paris 13 (physicochemical characterization). We thank the ImagoSeine facility (Jacques Monod Institute, Paris, France), and the France Biolmaging infrastructure supported by the French National
Research Agency (ANR-10-INSB-04, « Investmentsfit the future »). Parts of this work were supported by IPGP multidisciplinary program PARI, and by Paris-IdF region SESAME Grant no. 12015908.

\section{References}

1 P. Guardia, N. Pérez, A. Labarta and X. Batlle, Langmuir, 2010, 26, 5843-5847.

2 C. L. Ventola, P T Peer-Rev. J. Formul. Manag., 2017, 42, 742755.

3 A. Plan Sangnier, S. Preveral, A. Curcio, A. K. A. Silva, C. T. Lefèvre, D. Pignol, Y. Lalatonne and C. Wilhelm, J. Controlled Release, 2018, 279, 271-281.

4 H.-L. Xu, K.-L. Mao, Y.-P. Huang, J.-J. Yang, J. Xu, P.-P. Chen, Z.-L. Fan, S. Zou, Z.-Z. Gao, J.-Y. Yin, J. Xiao, C.-T. Lu, B.-L. Zhang and Y.-Z. Zhao, Nanoscale, 2016, 8, 14222-14236.

5 A. Tomitaka, H. Arami, Z. Huang, A. Raymond, E. Rodriguez, Y. Cai, M. Febo, Y. Takemura and M. Nair, Nanoscale, 2017, 10, 184-194.

6 S. Uthaman, M. Muthiah, I.-K. Park and C.-S. Cho, Polym Nanomater. Gene Ther., 2016, 215-230.

7 A. Espinosa, J. Kolosnjaj-Tabi, A. Abou-Hassn, A. Plan Sangnier, A. Curcio, A. K. A. Silva, R. Di Corato, S. Neveu, T. Pellegrino, L. M. Liz-Marzan and C. Wilhelm, Adv. Funct. Mat., 2018, 28, 1803660

8 E. Cazares-Cortes, S. Cabana, C. Boitard, E. Nehlig, N. Griffete, J. Fresnais, C. Wilhelm, A. Abou-Hassan and C. Ménager, Adv. Drug Deliv. Rev., 2019, 138, 233-246.

9 H. Wu, L. Song, L. Chen, Y. Huang, Y. Wu, F. Zang, Y. An, H. Lyu, M. Ma, J. Chen, N. Gu and Y. Zhang, Nanoscale, 2017, 9, 1617516182.

10 L. Accomasso, C. Gallina, V. Turinetto and C. Giachino, Stem Cells Int., 2016, 2016, 7920358.

11 R. Hachani, M. A. Birchall, M. W. Lowdell, G. Kasparis, L. D. Tung, B. B. Manshian, S. J. Soenen, W. Gsell, U. Himmelreich, C. A. Gharagouzloo, S. Sridhar and N. T. K. Thanh, Sci. Rep., 2017, 7, 7850.

12 A. V. Naumova, M. Modo, A. Moore, C. E. Murry and J. A. Frank, Nat. Biotechnol., 2014, 32, 804-818.

13 S. J. Kim, B. Lewis, M. Steiner, U. V. Bissa, C. Dose and J. A. Frank, Contrast Media Mol. Imaging, 2016, 11, 55-64.

14 V. Du, N. Luciani, S. Richard, G. Mary, C. Gay, F. Mazuel, M. Reffay, P. Menasché, O. Agbulut and C. Wilhelm, Nat. Commun., 2017, 8, 400 .

15 S. Ghosh, S. R. P. Kumar, I. K. Puri and S. Elankumaran, Cell Prolif., 2016, 49, 134-144.

16 W.-B. Shen, P. Anastasiadis, B. Nguyen, D. Yarnell, P. J. Yarowsky, V. Frenkel and P. S. Fishman, Cell Transplant., 2017, 26, 1235-1246.

17 D. Tukmachev, O. Lunov, V. Zablotskii, A. Dejneka, M. Babic, E. Syková and Š. Kubinová, Nanoscale, 2015, 7, 3954-3958.

18 H. Arami, A. Khandhar, D. Liggitt and K. M. Krishnan, Chem. Soc. Rev., 2015, 44, 8576-8607.

19 M. Marín-Barba, H. Gavilán, L. Gutiérrez, E. Lozano-Velasco, I. Rodríguez-Ramiro, G. N. Wheeler, C. J. Morris, M. P. Morales and A. Ruiz, Nanoscale, 2018, 10, 690-704.

20 M. Tadic, S. Kralj and L. Kopanja, Mater. Charact., 2019, 148, 123-133.

21 L. Guerrini, R. A. Alvarez-Puebla and N. Pazos-Perez, Mater., 2018, 11, 1154. 
22 A.-H. Lu, E. L. Salabas and F. Schüth, Angew. Chem. Int. Ed., 2007, 46, 1222-1244.

23 K. Andreas, R. Georgieva, M. Ladwig, S. Mueller, M. Notter, M. Sittinger and J. Ringe, Biomaterials, 2012, 33, 4515-4525.

24 A. T. Ngo and M. P. Pileni, J. Phys. Chem. B, 2001, 105, 53-58.

25 L. Li, K. Y. Mak, C. W. Leung, K. Y. Chan, W. K. Chan, W. Zhong and P. W. T. Pong, Microelectron. Eng., 2013, 110, 329-334.

26 E. Guénin, Y. Lalatonne, J. Bolley, I. Milosevic, C. Platas-Iglesias and L. Motte, J. Nanoparticle Res., 2014, 16, 2596.

27 M. Das, D. Mishra, P. Dhak, S. Gupta, T. K. Maiti, A. Basak and P. Pramanik, Small, 2009, 5, 2883-2893.

28 E. Amstad, T. Gillich, I. Bilecka, M. Textor and E. Reimhult, Nano Lett., 2009, 9, 4042-4048.

29 T. J. Daou, S. Buathong, D. Ung, B. Donnio, G. Pourroy, D. Guillon and S. Bégin, Sens. Actuators B Chem., 2007, 126, 159-162.

30 P. Daniel, S. I. Shylin, H. Lu, M. N. Tahir, M. Panthöfer, T. Weidner, A. Möller, V. Ksenofontov and W. Tremel, J. Mater. Chem. C, 2018, 6, 326-333.

31 J. Bolley, E. Guenin, N. Lievre, M. Lecouvey, M. Soussan, Y. Lalatonne and L. Motte, Langmuir, 2013, 29, 14639-14647.

32 J. Bolley, Y. Lalatonne, O. Haddad, D. Letourneur, M. Soussan, J. Pérard-Viret and L. Motte, Nanoscale, 2013, 5, 11478-11489.

33 E. Cheraghipour, S. Javadpour and A. R. Mehdizadeh, J. Biomed. Sci. Eng., 2012, 5, 715-719.

34 S. Richard, M. Boucher, A. Saric, A. Herbet, Y. Lalatonne, P. X. Petit, S. Mériaux, D. Boquet and L. Motte, J. Mater. Chem. B, 2017, 5, 2896-2907.

35 C. Wilhelm, F. Gazeau, J. Roger, J. N. Pons and J.-C. Bacri, Langmuir, 2002, 18, 8148-8155.

36 I. Lynch, T. Cedervall, M. Lundqvist, C. Cabaleiro-Lago, S. Linse and K. A. Dawson, Adv. Colloid Interface Sci., 2007, 134-135, 167-174.

37 I. Lynch and K. A. Dawson, Nano Today, 2008, 3, 40-47.

38 L. Treuel, X. Jiang and G. U. Nienhaus, J. R. Soc. Interface, 2013, 10, 20120939.

39 D. Walczyk, F. B. Bombelli, M. P. Monopoli, I. Lynch and K. A. Dawson, J. Am. Chem. Soc., 2010, 132, 5761-5768.

40 F. Wang, L. Yu, M. P. Monopoli, P. Sandin, E. Mahon, A. Salvati and K. A. Dawson, Nanomedicine Nanotechnol. Biol. Med., 2013, 9, 1159-1168.

41 S. R. Saptarshi, A. Duschl and A. L. Lopata, J. Nanobiotechnology, 2013, 11, 26.

42 L. Treuel, M. Malissek, J. S. Gebauer and R. Zellner, ChemPhysChem, 2010, 11, 3093-3099.

43 U. Sakulkhu, L. Maurizi, M. Mahmoudi, M. Motazacker, M. Vries, A. Gramoun, M.-G. O. Beuzelin, J.-P. Vallée, F. Rezaee and H. Hofmann, Nanoscale, 2014, 6, 11439-11450.

44 C. D. Walkey and W. C. W. Chan, Chem. Soc. Rev., 2012, 41, 2780-2799.

45 A. S. Arbab, L. B. Wilson, P. Ashari, E. K. Jordan, B. K. Lewis and J. A. Frank, NMR Biomed., 2005, 18, 383-389.

46 L. Gutiérrez, S. Romero, G. B. da Silva, R. Costo, M. D. Vargas, C. M. Ronconi, C. J. Serna, S. Veintemillas-Verdaguer and M. Del Puerto Morales, Biomed. Tech., 2015, 60, 417-425.

47 F. Mazuel, A. Espinosa, N. Luciani, M. Reffay, R. Le Borgne, L. Motte, K. Desboeufs, A. Michel, T. Pellegrino, Y. Lalatonne and C. Wilhelm, ACS Nano, 2016, 10, 7627-7638.

48 S. Mourdikoudis, R. M. Pallares and N. T. K. Thanh, Nanoscale, 2018, 10, 12871-12934.

49 A. Van de Walle, A. Plan Sangnier, A. Abou-Hassan, A. Curcio, M. Hémadi, N. Menguy, Y. Lalatonne, N. Luciani and C. Wilhelm, Proc. Natl. Acad. Sci. U. S. A., 2019, 116, 4044-4053.
50 F. Mazuel, A. Espinosa, G. Radtke, M. Bugnet, S. Neveu, Y. Lalatonne, G.A. Botton, A. Abou-Hassan and C. Wilhelm. Adv Funct. Mat., 2007, 27, 1605997.

51 S. Richard, V. Eder, G. Caputo, C. Journé, P. Ou, J. Bolley, L. Louedec, E. Guenin, L. Motte, N. Pinna and Y. Lalatonne, Nanomed., 2016, 11, 2769-2779.

52 D. Portet, B. Denizot, E. Rump, J.-J. Lejeune and P. Jallet, J. Colloid Interface Sci., 2001, 238, 37-42.

53 A. Quarta, A. Curcio, H. Kakwere and T. Pellegrino, Nanoscale, 2012, 4, 3319-3334.

54 C. Chouly, D. Pouliquen, I. Lucet, J. J. Jeune and P. Jallet, J. Microencapsul., 1996, 13, 245-255.

55 P. K. Thalla, A. Contreras-García, H. Fadlallah, J. Barrette, G. De Crescenzo, Y. Merhi and S. Lerouge, BioMed Res. Int., 2013, 2013, 962376.

56 O. J. G. M. Goor, J. E. P. Brouns and P. Y. W. Dankers, Polym. Chem., 2017, 8, 5228-5238.

57 F. Fuertges and A. Abuchowski, J. Controlled Release, 1990, 11, 139-148.

58 L. H. Reddy, J. L. Arias, J. Nicolas and P. Couvreur, Chem. Rev., 2012, 112, 5818-5878.

59 Y. Aray, M. Marquez, J. Rodríguez, D. Vega, Y. Simón-Manso, S. Coll, C. Gonzalez and D. A. Weitz, J. Phys. Chem. B, 2004, 108, 2418-2424.

60 V. B. Damodaran and N. S. Murthy, Biomater. Res., 2016, 20, 18. 61 S. Lüsse and K. Arnold, Macromolecules, 1996, 29, 4251-4257.

62 A. Ruiz, L. Gutiérrez, P. R. Cáceres-Vélez, D. Santos, S. B. Chaves, M. L. Fascineli, M. P. Garcia, R. B. Azevedo and M. P. Morales, Nanoscale, 2015, 7, 16321-16329.

63 T. L. Moore, L. Rodriguez-Lorenzo, V. Hirsch, S. Balog, D. Urban, C. Jud, B. Rothen-Rutishauser, M. Lattuada and A. Petri-Fink, Chem. Soc. Rev., 2015, 44, 6287-6305.

64 H. T. R. Wiogo, M. Lim, V. Bulmus, J. Yun and R. Amal, Langmuir, 2011, 27, 843-850.

65 Z.-G. Yue, W. Wei, P.-P. Lv, H. Yue, L.-Y. Wang, Z.-G. Su and G.-H. Ma, Biomacromolecules, 2011, 12, 2440-2446.

66 G. Jutz, P. van Rijn, B. Santos Miranda and A. Böker, Chem. Rev., 2015, 115, 1653-1701.

67 P. Arosio, R. Ingrassia and P. Cavadini, Biochim. Biophys. Acta, 2009, 1790, 589-599.

68 G. Zhao, F. Bou-Abdallah, P. Arosio, S. Levi, C. Janus-Chandler and N. D. Chasteen, Biochemistry, 2003, 42, 3142-3150.

69 G. Stepien, M. Moros, M. Pérez-Hernández, M. Monge, L. Gutiérrez, R. M. Fratila, M. de Las Heras, S. Menao Guillén, J. J. Puente Lanzarote, C. Solans, J. Pardo and J. M. de la Fuente, ACS Appl. Mater. Interfaces, 2018, 10, 4548-4560. 